\title{
When can gravity path-entangle two spatially superposed masses?
}

\author{
Chiara Marletto and Vlatko Vedral \\ Clarendon Laboratory, University of Oxford, Parks Road, Oxford OXI 3PU, United Kingdom; \\ Centre for Quantum Technologies, National University of Singapore, 3 Science Drive 2, \\ Singapore 117543, Singapore; \\ Department of Physics, National University of Singapore, 2 Science Drive 3, Singapore 117542, Singapore; \\ and ISI Foundation, Institute for Scientific Interchange, Via Chisola 5, 10126 Torino, Italy
}

(Received 11 April 2018; published 1 August 2018)

\begin{abstract}
An experimental test of quantum effects in gravity has recently been proposed, where the gravitational field's ability to entangle two masses is used as a witness of its quantum nature. Here, we discuss what existing models for coupled matter and gravity predict for this experiment. Collapse-type models, and also quantum field theory in curved spacetime, as well as various induced gravities, do not predict entanglement generation; they would, therefore, be ruled out as fundamental descriptions of gravity if entanglement were observed. Instead, local linearized quantum gravity models predict that the masses can become entangled. We analyze the mechanism by which entanglement is established in such models, modeling a gravityassisted two-qubit gate.
\end{abstract}

DOI: $10.1103 /$ PhysRevD.98.046001

Witnessing quantum effects in the gravitational field has traditionally been considered extremely hard, due to the weakness of the gravitational interaction. For instance, there have been claims that detecting a graviton is practically impossible $[1,2]$. The predictions from various models of quantum gravity, therefore, would be unobservable, thus making the quantization of the gravitational field itself questionable.

Recently, a different type of test has been introduced [3,4], which is not affected by such difficulties. Instead of measuring directly quanta of the gravitational field, the proposal is to test whether the gravitational field can entangle two masses each in a superposition of two different locations. The logic is that if gravity can generate entanglement between the two masses, then it must be quantum. By a system "being quantum" hereinafter we shall mean that in order to describe it one needs at least two variables that do not commute with one another. This is an indirect test, because the quantum features of the field are detected by measuring observables of two test masses only (rather than, for instance, detecting gravitons or fluctuations of spacetime). In the experiment, each mass is put into a superposition of two paths, e.g., via an interferometer. If the interferometers are close enough, the state of each mass can be significantly modified by interacting gravitationally with the other mass. According to how matter couples to gravity (see below for a discussion), different outcomes may occur. However, if at the output of the interferometers the two masses are entangled, one can conclude, via the argument in [3], that the gravitational field mediating entanglement must itself be quantum. That argument does not assume any specific dynamics [3], but it assumes that there is no instantaneous action at a distance between the two masses. As noted in [5], observing entanglement would confirm some type of nonclassicality in the field, in the form of there being at least two noncommuting variables, but it would not guarantee that the field is a fully fledged quantum system. For example, one of the two noncommuting variables might be not physically observable. With the advent of general relativity, the gravitational field has been understood as a spacetime metric. The proposed experiment tests therefore whether spacetime metric can be quantum, i.e., whether it can exist in quantum superpositions.

There are, at present, a number of proposed models for coupling matter with gravity. In this paper, we analyze which predictions they provide for the experiment in question. We restrict attention to three cases: the linearized canonical approaches [6], collapse-type models [7-9], and semiclassical gravity [10,11].

From our analysis, it emerges that the experiment, if entanglement were observed, would rule out collapse models and also all approaches that take the semiclassical description of gravity to be fundamental. This is because in such models the gravitational field is classical-it only has one observable, thus it would not be capable of entangling the masses. (It would still be possible, however, to regard the semiclassical approaches as viable approximative descriptions, in certain regimes). Linear quantum gravity (to which all the canonical approaches would have to reduce) would, on the other hand, be confirmed. Thus this experiment differs from previous experiments that couple a quantum system to gravity, such the COW and related 
experiments $[12,13]$, which are compatible with the gravitational field being classical. Using linearized quantum gravity, we also explain how the entanglement between the two masses is generated, by using the gravitational field as a third system that acts as a mediator between the two. We shall discuss specifically the amount of entanglement between the masses and the field, as well as the entanglement between the two masses.

Confining attention to the regime where Newtonian contributions are dominant suffices to our analysis. In this regime, the spacetime metric can be approximated by $d s^{2}=-\left(1-2 \xi(\mathbf{r}, t) / c^{2}\right) d t^{2}+d \mathbf{r}^{2}$, where $\xi(\mathbf{r}, t)$ may be time-dependent and propagates at a finite speed. (This is different from classical Newtonian gravity, where the gravitational potential admits instantaneous action at a distance and it is static). In this regime, the nonperturbative approaches to quantum gravity, such as loop quantum gravity [14] and string theory [15], agree with the predictions of the linearized approach; thus the proposed experiment would test their prediction, too, in this regime.

We first recall the logic of the experiment. Consider two equal masses $Q_{1}$ and $Q_{2}$ each in, say, two Mach-Zehnder interferometers, each located horizontally in the Earth's gravitational field (so that both masses are approximately subject to the same background field, which can therefore be neglected for the purpose of computing phase differences). Let one of the arms be indicated by 0 and the other by 1 . Each mass after the first beam splitter is in the state $\frac{1}{\sqrt{2}}(|0\rangle+|1\rangle)$. If one supposes that the masses on different paths interact via the gravitational field, different things can happen according to how matter and gravity couple. Broadly speaking, the superpositions might undergo some collapse or not; and if they do not, they may or may not become entangled.

We now consider the predictions from linear quantum gravity. For clarity, we first analyze a two-qubit entangling gate acting on the masses, where they interact separately with the gravitational field, but not directly with one another (thereby ruling out action at a distance). We shall model this by requiring that the Hamiltonian does not contain products of operators acting both on $Q_{1}$ and $Q_{2}$. We shall refer from now on to quantized degrees of freedom (d.o.f.) of the field that mediate the interaction with the masses. These d.o.f. can be thought of as the longitudinal and scalar d.o.f. that appear in the gauge of the GuptaBleuler formalism, see [16].

We can model the gravitational field as a single quantum harmonic oscillator. In linear quantum gravity, $a$ and $a^{\dagger}$ can be interpreted as the bosonic annihilation and creation operators for gravitons. The two masses can be initially modeled as two qubits-whose z-component represents a discretized position of each mass-in this case, one of the two paths in an interferometer. Its eigenstates $|a\rangle$ where $a \in\{0,1\}$ represent the situation where the mass is on a definite path $a$; while $|a b\rangle$ describes the situation where the first mass is on path $a$ and the second on path $b$.
We shall now analyze how the relative phases in the quantum superpositions of masses are established thus inducing entanglement. First, the masses get entangled to the field, then the phases are generated through a generalized controlled-phase gate. Immediately after the action of the first beam splitter, the state of the two masses and the field is $\left|\phi_{0}\right\rangle=\frac{1}{2} \sum_{a, b \in\{0,1\}}|a b\rangle|\alpha\rangle$, where $|\alpha\rangle=$ $\mathrm{e}^{-\frac{1}{2}|\alpha|^{2}} \exp \left(\alpha\left(a^{\dagger}-a\right)\right)|0\rangle$ is a coherent state representing the spatial modes of gravity-possibly a continuum.

The two masses and the field then evolve into the state

$$
\left|\phi_{E 1}\right\rangle=U_{1}\left|\phi_{0}\right\rangle=\frac{1}{2} \sum_{a, b \in\{0,1\}}|a b\rangle\left|\alpha_{a, b}\right\rangle,
$$

where $\quad U_{1} \doteq \sum_{a, b \in\{0,1\}} P_{a b} \otimes D\left(\xi_{a b}\right) \quad$ and $\quad\left|\alpha_{a, b}\right\rangle=$ $\left|\alpha+i \sqrt{\xi_{a b}}\right\rangle=D\left(\xi_{a, b}\right)|\alpha\rangle$; we have defined the displacement operator as $D\left(\xi_{a, b}\right)=\exp \left(i \sqrt{\xi_{a, b}}\left(a^{\dagger}-a\right)\right)$ with $\sqrt{\xi_{a b}}$ being a real-numbered shift that depends on the coupling between the field and the masses, that brings about the desired phase-shift $\phi_{a, b}$ at the end (see below for more details). We have also defined the projectors $P_{a b}=$ $P_{a} \otimes P_{b}$, with $P_{0,1}=\frac{\left(i d \pm \sigma_{z}\right)}{2}$ being the projector operator for the location of each mass. We have also assumed that establishing the entanglement between the field and the masses takes place on time-scales much faster than the process that transfers the phase $\xi_{a, b}$ back from the field to the masses, evolving their composite system to the state $\left|\phi_{E 2}\right\rangle=U_{2}\left|\phi_{E 1}\right\rangle \approx \frac{1}{2} \sum_{a, b \in\{0,1\}} \exp \left(i \phi_{a, b}\right)|a b\rangle\left|\alpha_{a, b}\right\rangle$, where $U_{2}=\exp \left(w\left(a^{\dagger} a\right)\right), w$ is some real number with the property that $w \xi_{a, b}=\phi_{a, b}$, and, for the sake of this simple illustration, we have assumed to be in the regime where $|\alpha|$ is large and real (later, the full linearized model will present the exact solution for any coherent state). Finally, the interaction $U_{1}^{\dagger}$ between the field and the masses brings the field back to its original state and the masses remain entangled (to a degree depending on the phase): $\frac{1}{2} \sum_{a, b \in\{0,1\}} \exp \left(i \phi_{a, b}\right)|a b\rangle|\alpha\rangle$.

The key fact is that the above process relies on two complementary observables of the field, because the observables $\frac{1}{2 i}\left(a-a^{\dagger}\right)$ and $a^{\dagger} a$ are needed to generate the unitaries $U_{1}$ and $U_{2}$. The entanglement between the field and masses can be quantified by the reduced entropy of the masses. Since the field and the masses are weakly entangled, a good approximation of the reduced entropy is the linear entropy $S_{L}=1-\operatorname{Tr}\left(\rho_{\mathrm{Q}_{\mathrm{i}}}^{2}\right)$, where $\rho_{Q_{i}}$ is the reduced state of mass $Q_{i}$. The magnitude of the reduced entropy is given by one minus the overlap between the two gravitational states squared as in: $1-\left|\left\langle\alpha_{a b} \mid \alpha\right\rangle\right|^{2}=1-\exp \left(-\xi_{a b}\right) \approx \xi_{a b}$. This quantity could be very small compared to one, while still generate the desired entanglement between the two masses. Assuming the regime where the Newtonian contribution only is relevant, one has: $\phi_{a b}=w \alpha_{a b}=\frac{G m^{2}}{\hbar d_{a b}} \Delta t=$ $\left(\frac{m}{m_{P}}\right)^{2} \frac{c}{d_{a b}} \Delta t$, where $\Delta t$ is the interaction time between the 
two masses, $m_{P}$ is Planck's mass, $\mathrm{G}$ is the gravitational constant, and $d_{a b}$ is the distance between the position $a$ of the first mass and position $b$ of the second mass. One can identify $\alpha_{a b}=\left(\frac{m}{m_{P}}\right)^{2}$. The entanglement between a spatially superposed mass and the gravitational field (if, indeed, it is quantum) would then offer another way of understanding the Planck mass. Namely, if we really want a spatially superposed mass to entangle appreciably to the surrounding gravitational field, according to the above formula, we need to engage masses on the order of and larger than the Planck mass. The full linearized model, to be presented below, leads to the same conclusion.

Supposing that only the closer arms of the interferometers (labeled as 1) interact, the only phase present in the state of the masses before they enter their respective final beam splitter is $\phi_{11}$. In each of the interferometers, the probability for the mass to emerge on path 0 is $p_{0}=$ $\frac{1}{2}\left(1+\cos ^{2} \frac{\phi_{11}}{2}\right)$ (and $\left.p_{1}=1-p_{0}\right)$. When the two masses are maximally entangled we have $p_{0}=p_{1}=\frac{1}{2}$. This happens when $\phi_{11}=\pi$. On the other hand, when the two masses are not entangled, they separately undergo an ordinary interference: that happens when $\phi_{11}=2 n \pi$. In that case, each mass emerges on path 0 of the interferometer. For a fixed mass, by varying the arms' distance or their length, it is in principle possible to interpolate between those two cases, thus demonstrating entanglement. To confirm entanglement, one would require to measure two complementary observables on each interferometer. For example, consider the maximally entangled state $|0\rangle|+\rangle+|1\rangle|-\rangle$. If we measure the path of the first particle (the effective Pauli $\mathrm{Z}$ measurement), the second mass interferes with either the plus or the minus phase (i.e., it is in an eigenstate of the Pauli X). If, on the other hand, the second mass is first measured in $\mathrm{X}$, there is no interference of the first mass (meaning that $X_{1}$ and $X_{2}$ ) are not correlated. Therefore, the observable $X_{1} Z_{2}+Z_{1} X_{2}$ will suffice to witness entanglement.

We now compute the phases in the proposed experiment using the full linearized Hamiltonian, whose evolution can be approximated in discrete steps by the gate model presented above. This is obtained from the general linearized Hamiltonian, [2]: $H_{\text {int }}^{G}=-\frac{1}{2} h_{\mu \nu} T^{\mu \nu}$, where $T^{\mu \nu}$ is the stress-energy tensor and $h_{\mu \nu}$ is the perturbation of the metric tensor $g_{\mu \nu}$ away from the flat (Minkowski) spacetime. The quantized gravitational field is then written in terms of the graviton creation and annihilation operators $a(k, \sigma)$, $a^{\dagger}(k, \sigma)$, as

$$
h_{\mu \nu} \propto \sum_{\sigma} \int \frac{d^{3} k}{\sqrt{\omega_{k}}}\left\{a(k, \sigma) \epsilon_{\mu \nu}(k, \sigma) e^{i k_{\lambda} x^{\lambda}}+\text { H.c. }\right\},
$$

where $\epsilon_{\mu \nu}$ is the polarization tensor, $\sigma$ indicates two nonvanishing gravitational polarizations, while $\omega_{k}$ and $k$ represent the frequency and wave number of the mode, respectively (we are using the Einstein's convention of summation).

In our experiment, the masses are nonrelativistic and the stress-energy tensor would simplify to $T_{00}=m$. We can also consider, for simplicity, a single polarization and a discrete sum over the relevant gravitational quantum modes. The total Hamiltonian involving two masses and the gravitational field is, therefore,

$$
\begin{aligned}
H= & m c^{2}\left(b_{1}^{\dagger} b_{1}+b_{2}^{\dagger} b_{2}\right)+\sum_{k} \hbar \omega_{k} a_{k}^{\dagger} a_{k} \\
& -\sum_{k, n \in\{1,2\}} \hbar g_{k} b_{n}^{\dagger} b_{n}\left(a_{k} e^{i k x_{n}}+a_{k}^{\dagger} e^{-i k x_{n}}\right),
\end{aligned}
$$

where the first two terms are the free Hamiltonians of the masses and the field, respectively. We assume that the gravitation-matter coupling constant is given by $g_{k}=$ $m c \sqrt{\frac{2 \pi G}{\hbar \omega_{k} V}}$, where $V$ is the relevant volume of quantization (which will not feature in the relevant observables). The evolution of two masses of value $m$ at positions $x_{1}$ and $x_{2}$ interacting with the initial gravitational vacuum state can be solved exactly: $e^{i H t}|m\rangle|m\rangle|0\rangle=$ $\exp \left\{\hbar \sum_{k} V(k) t\right\}|m\rangle|m\rangle\left|\sum_{k} \frac{g}{\omega_{k}}\left(e^{-i k x_{1}}+e^{i k x_{2}}\right)\right\rangle$, where $V(k)=\frac{g_{k}^{2}}{2 \omega_{k}}\left(1+2 \cos \left(-i k\left(x_{2}-x_{1}\right)\right)\right)$.

When acting on the initially superposed state, this generates entanglement between the two masses by implementing the controlled-phase gate $U_{2} U_{1}$ described in the previous section. Note that only the position-dependent part of $V(k)$ contribute to the phase difference. The continuum version of the position-dependent part of $V(k)$, obtained by replacing the sum over $k$ by an integral, is

$$
\operatorname{Re}\left\{V \int d k \frac{4 \pi G m^{2}}{\hbar k^{2} V} e^{-i k\left(x_{1}-x_{2}\right)}\right\}=\frac{G m^{2}}{\hbar\left(x_{2}-x_{1}\right)},
$$

which gives us the same phase as described in the gate model.

We can assume that the interaction between the masses and the field is 'elastic', i.e., when the two masses are brought back to their original state, where each one of their positions is sharp, the field goes back to the original state, and it is unentangled with the masses. However, even if the interaction were not perfectly elastic, since the entanglement between the field and the masses is very small (for masses below Planck mass), as computed earlier, the state of the two masses is approximately not entangled with the field at the end, thus leaving the field approximately unchanged. The same result can be obtained with the usual Lagrangian formulation of quantum field theory, where the interaction is established via the exchange of a single graviton between the two masses and the field.

Observing entanglement would on the other hand refute all those theories which regard a semiclassical description 
of gravity as fundamental, [10,11]. In these theories, the background spacetime is classical, but the back-action of the masses prepared in some quantum state on the field can be taken into account as an average of the energymomentum tensor in the quantum state of the masses. The Einstein's equation reads: $R_{\mu \nu}-\frac{1}{2} R g_{\mu \nu}=8 \pi G\left\langle T_{\mu \nu}\right\rangle$, where $R_{\mu \nu}$ is the Ricci tensor; $R$ is its trace; and $g_{\mu \nu}$ is the metric tensor.

These theories provide powerful predictions such as the Unruh effect and the Hawking radiation, [11]. Yet, they cannot adequately describe quantum effects in gravity at the fundamental level, as already pointed out in [17]. This is because they resort to a field which is classical-in the sense that it has no pair of noncommuting observables. The field strength at each point is determined by an average of the stress-energy tensor in the quantum state of the masses. In the situation of our experiment, each mass would, therefore, be affected by the average of the gravitational field generated by the other superposed mass. Supposing each mass is initially in an equally-weighted superposition of the two possible locations, each mass would experience the potential generated by the other mass $m$ positioned at a distance which is the average of the position of the other mass in its quantum state. Assuming once more that only the gravitational interaction affects the branch corresponding to the arm of the interferometer closer to the other (labeled by 1), the state of the mass would become $\frac{1}{\sqrt{2}}\left(|0\rangle+e^{i \phi_{m}}|1\rangle\right)$, where $\phi_{m}=G \frac{m^{2} t}{\hbar d_{m}}$ where $d_{m}=\frac{d_{1}+d_{2}}{2}$, and $d_{1}$ and $d_{2}$ are, respectively, the distances of path 1 of one interferometer from paths 0 and 1 of the other. Likewise, by symmetry, for the other mass. Thus the phase acquired would be a local phase, which cannot generate entanglement between the masses. Each mass would be undergoing a separate, COW-type experiment: the state of the two masses would be at any time a product state. Semiclassical theories would, therefore, be refuted as fundamental descriptions of gravity by observing entanglement in the proposed experiment. The same prediction of no entanglement would be reached by models that resort the (nonequivalent) procedure of averaging the linearized quantum gravity Hamiltonian in the quantum state of the two masses. This would also provide only local phases (albeit different from the former case).

The other class of theories that would be ruled out by witnessing entanglement are collapse-type models predicting a collapse of the mass wave function at the experiment's scales[7-9], i.e., an irreversible transition to a state where the position is sharp. Consider for example the decoherence time a mass of $10^{-12} \mathrm{~kg}$ superposed across two different locations, approximately $10^{-4} \mathrm{~m}$ apart, (the spatial extent of each interferometer). According to Penrose's collapse models, [8], this time is of the order $t=\frac{\hbar}{G \frac{m^{2}}{d}} \approx 10^{-13} \mathrm{~s}$, well below $10^{-6} \mathrm{~s}$, required for our experiment. There is a subtle difference between collapse occurring, and decoherence happening while an interference experiment is taking place. For example, in neutron interferometry, a neutron spin couples to neighboring spins and affects their state. Since the neutron is in a superposition of two spatial locations, the neighboring spins are entangled with the spatial states of the neutron. However, when the two arms of the interferometer are recombined to measure the interference the two environmental states effectively become the same. This is why, despite the neutron having been entangled with environmental spins inside the interferometer, interference is still observable. A massive superposition can also become entangled with the gravitational field, as explained, and still evolve coherently. This decoherence could be discriminated from the genuine collapse. We can also discriminate de-phasing due to gravity from the gravitationally induced collapse, because the former would permit generating entanglement, but the latter would not. However, we cannot discriminate gravitationally induced spontaneous emission (still a fully quantum effect due to the vacuum state of the gravitational field) from a collapse, as in both cases interference would not occur.

Finally, another approach $[18,19]$ treats gravity as an induced field by the quantum vacuum fluctuations of all other fields. According to this logic, gravity is not a fundamental force and, therefore, does not need to be quantized. This approach, treating the gravitational field as classical, are not able to account for entanglement generation in our experiment. Still, it may be possible that entanglement generation is caused by other quantum fields, leading to an effective gravitational phase. This remains an open question.

\section{ACKNOWLEDGMENTS}

C. M. thanks the Templeton World Charity Foundation and the Eutopia Foundation. V. V. thanks the National Research Foundation, Prime Minister's Office, Singapore, under its Competitive Research Programme (CRP Grant No. NRF-CRP14-2014-02) and administered by Centre for Quantum Technologies, National University of Singapore. C. M. and V. V. thank the anonymous referees for insightful and helpful comments. 
[1] F. Dyson, Int. J. Mod. Phys. A 28, 1330041 (2013).

[2] S. Boughn and T. Rothman, Classical Quantum Gravity 23, 5839 (2006); T. Rothman and S. Boughn, Found. Phys. 36, 1801 (2006).

[3] C. Marletto and V. Vedral, Phys. Rev. Lett. 119, 240402 (2017).

[4] S. Bose, A. Mazumdar, G. W. Morley, H. Ulbricht, M. Toroš, M. Paternostro, A. A. Geraci, P. F. Barker, M. S. Kim, and G. Milburn, Phys. Rev. Lett. 119, 240401 (2017).

[5] M. Reginatto and M. Hall, J Phys A 51, 085303 (2018).

[6] C. Kiefer, Quantum Gravity (Clarendon Press, Oxford, 2004).

[7] L. Diosi, Phys. Lett. A 120, 377 (1987); L. Diosi, Phys. Rev. A 40, 1165 (1989).

[8] R. Penrose, Gen. Relativ. Gravit. 28, 581 (1996); R. Penrose, Phil. Trans. R. Soc. A 356, 1927 (1998).

[9] G. C. Ghirardi, A. Rimini, and T. Weber, Phys. Rev. D 34, 470 (1986).

[10] B. DeWitt, Phys. Rep. 19, 295 (1975).
[11] N. D. Birrell and P.C.W. Davies, Quantum Fields in Curved Space (Cambridge University Press, Cambridge, England, 1982), Chap. 6.

[12] R. Colella, A. W. Overhauser, and S. A. Werner, Phys. Rev. Lett. 34, 1472 (1975).

[13] I. Pikovski, M. Zych, F. M. Costa, and Č. Brukner, Nat. Phys. 11 (2015).

[14] C. Rovelli, Living Rev. Relativity 1, 1 (1998).

[15] B. Zwiebach, A First Course in String Theory (Cambridge University Press, Cambridge, England, 2009).

[16] S. Gupta, Proc. Phys. Soc. A 65, 608 (1952).

[17] D. Page and C. D. Geilker, Phys. Rev. Lett. 47, 979 (1981).

[18] A. D. Sakharov, Dokl. Akad. Nauk SSSR. Ser. Fiz. 177, 70 (1967) [Sov. Phys. Dokl. 12, 1040 (1968)]; reprinted in: A. D. Sakharov, Usp. Fiz. Nauk 161, 64 (1991) [Sov. Phys. Usp. 34, 394 (1991)]; Gen. Relativ. Gravit. 32, 365 (2000).

[19] M. Visser, Mod. Phys. Lett. A 17, 977 (2002). 